\title{
Fine Tuning of Resonances and Periodic Solutions of Hamiltonian Systems Near Equilibrium
}

\author{
G. F. Dell'Antonio* \\ Dipartimento di Matematica, Università di Roma, La Sapienza, Piazzale Aldo Moro 2, I-00185 Roma, \\ Italy
}

\section{Introduction}

Consider in $R^{2 n}$ a Hamiltonian System which has the origin as isolated equilibrium. We use the notation $z_{k} \equiv\left(q_{k}, p_{k}\right), k=i \cdots n$, and we assume that the Hamiltonian $H$ is of class $C^{2}$ and that $H_{2}$, the quadratic part of $H$, is given by

$$
H_{2}=1 / 2 \sum_{0}^{n} v_{i} z_{i}^{2}, \quad z_{i}^{2}=q_{i}^{2}+p_{i}^{2},
$$

with $v_{i} \neq 0, i=1 \cdots n$. If $v_{i} \cdot v_{j}^{-1}$ is not an integer for any pair $i, j(i \neq j)$ a theorem by Lyapunov [1] states that one can find a neighborhood $N$ of the origin such that in $N$ there are $n$ families of elliptic periodic solutions of (1.1); the $k$ th family has minimal periods approximately equal to $2 \pi v_{k}^{-1}$ and lies approximately on the hyperplane $\left\{z \mid z_{h}=0\right.$ if $\left.h \neq k\right\}$. One can use as parameter the distance from the origin. These results follow from an application of the Inverse Function Theorem to the periodicity condition.

General lower bounds on the number of periodic solutions on every energy surface sufficiently close to the origin have been obtained within a variational approach. These results hold independently of whether there is a resonance among the frequencies, but are often restricted to the case when $\mathrm{H}_{2}$ is of definite sign [2] (see however [6]); also, the localization and stability of the periodic solutions are in general not known.

On the other hand, if $v_{i}=k v_{j}$ for some $i \neq j, k \in \mathbf{Z}$ (a resonant case) the number of families can be different from the one indicated in Lyapunov's Theorem, and some of the periodic solutions are hyperbolic. Systems with resonance have been studied extensively in phase space [3]. [4] leading to various estimates on the number of families of periodic solutions and their stability. In particular it was shown (see also [5]) that, if $n=2, v_{2}=2 v_{1}$, one has in general three families; the minimal period is approximately $2 \pi v_{1}^{-1}$ for two of them, while it is approximately

\footnotetext{
$\star$ C.N.R., GNFM
} 
equal to $2 \pi v_{2}^{-1}$ for the third. If one considers instead the case $v_{2}=-2 v_{1}$, one has in general only one family, with minimal period approximately $2 \pi v_{2}^{-1}$.

In view of these results, it is natural to ask whether there is a discontinuity in the behaviour of the system when the set of frequencies in $\mathrm{H}_{2}$ approaches a resonance. To answer this question, we shall study the system in a full neighborhood of a resonance, i.e. in a domain in $R^{2 n} \times R^{n}$ of the form

$$
N=\left\{(z, v) \mid z \in \mathbf{B}_{\varepsilon}^{2 n}, v-v_{0} \in\left(\mathbf{B}_{\alpha(\varepsilon)}^{n}\right)\right\},
$$

where $v_{0}$ is a set of resonant frequencies, $\alpha(\varepsilon)=\varepsilon^{\alpha}$ for some $\alpha>0$ to be chosen later, and $\mathbf{B}_{\varepsilon} \equiv\left\{x \in R^{2 n},|x| \leqq \varepsilon\right\}$.

Several aspects of this "detuning" (or bifurcation) have been studied previously by J. Duistermaat [5] using the variational approach. Many of our results overlap with those of [5], and we refer to that paper for a list of previous results in various cases of resonance.

Our aim is to give a systematic treatment within phase space; this approach, as compared to the variational one, has the advantage that one can exploit symplectic transformations, through the use of normal forms. At the same time one can apply directly in our context the estimates which have been derived within the variational approach through the use of a cohomological index [6].

We shall in fact prove, in the phase space approach, that the number of families of periodic solutions near the origin is linked to the number of critical points of a suitable function $F$ defined on a manifold in phase space.

The function $F$ and the manifold are closely related to the ones introduced in [6] through a reduction to a finite dimensional subspace of functions. On the other hand, the results obtained in the phase-space approach hold only "generically" (in a sense to be made precise later) since to exploit the Inverse Function Theorem one needs transversality conditions which are stronger than those required in the variational approach.

A basic role in the analysis described here is held by a lemma on commuting vector fields (Lemma 1) which may have an independent interest and is therefore formulated and proved in greater generality than needed in the rest of the paper.

The paper is organized as follows: In Sect. 2 we prove the lemma and some related results. In Sect. 3 we specialize to the case of Hamiltonian vector fields, using the theory of normal forms. Here we connect the families of periodic solutions to the critical points of a suitable function in phase space. We also discuss the stability of these families of solutions. In Sect. 4 we discuss the detuning, and, as an example, we give a detailed analysis of the cases $n=2, v_{2}=2 v_{1}, v_{2}=-2 v_{1}$, $2 v_{2}= \pm 3 v_{1}$.

\section{A Lemma on Commuting Vector Fields}

We prove first a simple lemma which plays a central role in what follows. Consider in $R^{2 n}$ the vector field

$$
Y_{\varepsilon}(z)=A z+\varepsilon G(z, \varepsilon), \quad[G(z, \varepsilon), A z]_{\mathrm{Lie}}=0,
$$

where $G$ is Lipshitz-continuous in $z$ in $B_{a}=\{z \mid z \leqq a\}$, uniformly for $0 \leqq \varepsilon \leqq \varepsilon_{1}$. The 
matrix $A$ is antisymmetric with eigenvalues $\pm i v_{1} \cdots \pm i v_{n}$, which satisfy

$$
v_{k}=s_{k} \underline{\underline{v}}, \quad s_{k} \in \mathbf{Z} /\{0\}, \quad k=1 \cdots n, \quad \underline{v} \neq 0 .
$$

We shall use the notation

$$
\tau_{k} \equiv 2 \pi\left|v_{k}\right|^{-1}, \quad \underline{\tau} \equiv 2 \pi|\underline{v}|^{-1},
$$

and denote by $\tau$ the largest positive number for which

$$
\tau_{k}=m_{k} \tau, \quad m_{k} \in \mathbf{Z}
$$

One has then

$$
\underline{\tau}=N \tau, \quad N=m_{k}\left|s_{k}\right| \quad \forall k .
$$

For $\varepsilon \geqq 0$, we denote by $\varphi_{\varepsilon}\left(t, z^{*}\right)$ the solution of

$$
d z / d t=Y_{\varepsilon}(z), \quad \varphi_{\varepsilon}\left(0, z^{*}\right)=z^{*} .
$$

Notice the following: if for given $\varepsilon, z^{*}$ there exists $\lambda \in R$ such that

$$
G\left(z^{*}, \varepsilon\right)=\lambda A z^{*}
$$

then this relation holds also for $\varepsilon, \varphi_{0}\left(t, z^{*}\right) \forall t$, since the two vector fields $A z$ and $G(z, \varepsilon)$ commute. Therefore

$$
\varphi_{\varepsilon}\left(t . z^{*}\right)=\varphi_{0}\left((1+\lambda)^{-1} t, z^{*}\right)
$$

so that in particular $\varphi_{\varepsilon}\left(t, z^{*}\right)$ is periodic with minimal period

$$
T_{\varepsilon}\left(z^{*}\right)=(1+\lambda) T_{0}\left(z^{*}\right) .
$$

We now prove (Lemma 2.1) that for every $T>0$, if $\varepsilon$ is small enough, all periodic solutions of (2.4) arise in this way, i.e. have the form (2.6).

It follows from (2.2) that for every $z^{*}$ in $R^{2 n}, \varphi_{0}\left(t, z^{*}\right)$ is periodic. We denote by $T_{0}\left(z^{*}\right)$ its minimal period and by $K\left(z^{*}\right)$ the positive integer for which

$$
T_{0}\left(z^{*}\right)=K\left(z^{*}\right) \tau \text {. }
$$

From the definition of $\varphi_{0}$ one easily verifies that given $c_{2}>0$, one can find $\varepsilon_{0}>0$, and $c_{1}>0$, depending on $a$ and on $v_{1} \cdots v_{n}$, such that for each $z^{*} \in \mathbf{B}_{a}, 0<\varepsilon<\varepsilon_{0}$, $t \in R$ there exists an integer $m\left(z^{*}, \varepsilon, t\right)$ with the property that

$$
\left|\varphi_{0}\left(t, z^{*}\right)-z^{*}\right|<c_{1} \varepsilon \quad \text { implies }\left|t-m\left(z^{*}, \varepsilon, t\right) \tau\right|<c_{2} \varepsilon .
$$

If $\varphi_{\varepsilon}\left(t, z^{*}\right)$ is periodic, we shall denote by $T_{\varepsilon}\left(z^{*}\right)$ its minimal period. Let $T$ be an arbitrary but fixed positive number; we shall look for periodic solutions of (2.3) for which $T_{\varepsilon}\left(z^{*}\right)<T$. If $\varphi_{\varepsilon}\left(t, z^{*}\right)$ is periodic, and $\left|z^{*}\right|<a$, one can find $c_{1}>0$ such that

$$
\left|z^{*}-\varphi_{0}\left(T_{\varepsilon}\left(z^{*}\right), z^{*}\right)\right|=\left|\varphi_{\varepsilon}\left(T_{\varepsilon}\left(z^{*}\right), z^{*}\right)-\varphi_{0}\left(T_{\varepsilon}\left(z^{*}\right), z^{*}\right)\right|<c_{1} \varepsilon .
$$

We conclude from (2.8), (2.9) that for all $0<\varepsilon<\varepsilon_{0}$, for all $z^{*} \in \mathbf{B}_{a}$ for which $\varphi_{\varepsilon}\left(t, z^{*}\right)$ is periodic one can find an integer $K$ such that

$$
\left|K T_{\varepsilon}\left(z^{*}\right)-\underline{\tau}\right|<c_{2} \varepsilon .
$$


Define $b(a)$ to be the largest positive number for which

$$
\left|z^{*}\right|<b(a) \Rightarrow\left|\varphi_{0}\left(t, z^{*}\right)\right|<a \quad \forall \varepsilon \in\left[0, \varepsilon_{0}\right] \forall t \in\left[0, T_{\varepsilon}\left(z^{*}\right)\right] .
$$

One can now prove

Lemma 2.1. Let $Y_{\varepsilon}(z)$ be given as in (2.1), (2.2). Given $T>0$, one can find $\gamma>0$ with the property that, if $\varepsilon<\gamma T^{-1}$ and $z^{*} \in \mathbf{B}_{b(a)}$, then $\varphi_{\varepsilon}\left(t, z^{*}\right)$ is periodic with minimal period $T_{\varepsilon}\left(z^{*}\right)<T$ if and only if there exists $\lambda\left(z^{*}, \varepsilon\right) \in R$ such that (2.5) holds.

When (2.5) holds, $\varphi_{\varepsilon}\left(t, z^{*}\right)$ coincides with $\varphi_{0}\left(t, z^{*}\right)$ up to a time scale,

Proof. For fixed $z^{*}, \varepsilon$ define $\lambda$ as follows:

$$
1+\varepsilon \lambda=\underline{\tau} / K T_{\varepsilon}\left(z^{*}\right),
$$

so that, from (2.4), (2.10)

$$
\exp \left\{K(1+\varepsilon \lambda) T_{\varepsilon}\left(z^{*}\right) A\right\}=\mathrm{I}
$$

Define $\psi_{\varepsilon}\left(t, z^{*}\right)$ by

$$
\psi_{\varepsilon}\left(t, z^{*}\right) \equiv \exp [-K(1+\varepsilon \lambda) t A] \varphi_{\varepsilon}\left(t, z^{*}\right) .
$$

It follows from (2.11) that $\psi_{\varepsilon}\left(t, z^{*}\right)$ is periodic with period $K T_{\varepsilon}\left(z^{*}\right)$. Differentiating (2.12) and using (2.3) one concludes that $\psi_{\varepsilon}\left(t, z^{*}\right)$ is the solution of

$$
d \psi_{\varepsilon} / d t=\varepsilon \Theta\left(\psi_{\varepsilon}, \varepsilon\right) \quad \psi_{\varepsilon}\left(0, z^{*}\right)=z^{*}
$$

where

$$
\Theta(z, \varepsilon) \equiv \exp [-K(1+\varepsilon \lambda) t A]\left\{G\left(\exp [K(1+\varepsilon \lambda) t A] \varphi_{\varepsilon}(t, z), \varepsilon\right)-\lambda A z\right\}=G(z, \varepsilon)-\lambda A z .
$$

The last equality in (2.13) follows from the fact that the fields $G(z, \varepsilon)$ and $A z$ commute.

Without loss of generality we can assume that

$$
\left|\Theta\left(\psi_{\varepsilon}\left(t, z^{*}\right), \varepsilon\right)\right| \leqq\left|\Theta\left(z^{*}, \varepsilon\right)\right| \quad \forall 0 \leqq t \leqq T_{\varepsilon}\left(z^{*}\right),
$$

so that

$$
\left|\psi_{\varepsilon}\left(t, z^{*}\right)\right| \leqq \varepsilon t\left|\Theta\left(z^{*}, \varepsilon\right)\right| \quad \forall 0 \leqq t \leqq T_{\varepsilon}\left(z^{*}\right) .
$$

Since $\psi_{\varepsilon}$ is periodic

$$
0=\int_{0}^{K T \varepsilon\left(z^{*}\right)} \Theta\left(\psi_{\varepsilon}\left(t, z^{*}\right), \varepsilon\right) d t=K T_{\varepsilon}\left(z^{*}\right) \Theta\left(z^{*}, \varepsilon\right)+\int_{0}^{K T \varepsilon\left(z^{*}\right)}\left[\Theta\left(\psi_{\varepsilon}\left(t, z^{*}\right), \varepsilon\right)-\Theta\left(z^{*}, \varepsilon\right)\right] d t,
$$

so that, using (2.14) and the Lipshitz condition on $G$

$$
K T_{\varepsilon}\left(z^{*}\right)\left|\Theta\left(z^{*}, \varepsilon\right)\right| \leqq c_{4} \int_{0}^{K T \varepsilon\left(z^{*}\right)}\left|\psi_{\varepsilon}\left(t, z^{*}\right)-z^{*}\right| d t \leqq 1 / 2 c_{4} \varepsilon K^{2} T_{\varepsilon}^{2}\left(z^{*}\right)\left|\Theta\left(z^{*}, \varepsilon\right)\right|,
$$

i.e., if $\left|\Theta\left(z^{*}, \varepsilon\right)\right| \neq 0$,

$$
2 \leqq \varepsilon c_{4} K T_{\varepsilon}\left(z^{*}\right)
$$

We conclude that, if $\varepsilon \leqq \min \left\{\varepsilon_{0}, 2\left[c_{4} K T\right]^{-1}\right\}$, and if $T_{\varepsilon}\left(z^{*}\right) \leqq T$, one must have 
$\left|\Theta\left(z^{*}, \varepsilon\right)\right|=0$, i.e. $G\left(z^{*}, \varepsilon\right)=\lambda A z^{*}$. In particular

$$
T_{\varepsilon}\left(z^{*}\right)=(1+\lambda \varepsilon)^{-1} T_{0}\left(z^{*}\right)
$$

so that

$$
T_{0}\left(z^{*}\right)=\underline{\tau} \quad \Rightarrow \quad K=1 .
$$

Conversely, if (2.5) holds, then one has

$$
G\left(\varphi_{0}\left(t, z^{*}\right), \varepsilon\right)=\lambda\left(z^{*}, \varepsilon\right) A\left(t, z^{*}\right),
$$

since $G(z, \varepsilon)$ and $A z$ commute. Therefore $Y_{\varepsilon}(z)$ is parallel to $A z$ along the orbit of $\varphi_{0}$ starting at $z^{*}$, and $\varphi_{0}\left(t, z^{*}\right)$ solves the equation

$$
d z / d t=(1+\varepsilon \lambda)^{-1} Y_{\varepsilon}(z)
$$

The remaining statement in Lemma 2.1 is obvious.

Remark 2.2. Condition (2.10) can be written in the form

$$
\lambda \nabla(A z, A z)=-2 A G(z, \varepsilon) .
$$

Let

$$
\Sigma_{c} \equiv\{z \mid(A z, A z)=c\} .
$$

Then (2.17) is equivalent to the condition that $z$ be a critical point for $(A G)_{0}(z)$, the projection of $A G$ to $T \Sigma_{c}(z)$.

The flow $t \rightarrow \exp \{A t\}$ provides a natural $S^{1}$ action on $(A G)_{0}$ and on the (compact) manifold $\Sigma_{c}$. A lower bound on the number of critical orbits for $(A G)_{0}$ (and therefore on the number of periodic solutions of (2.4) with period smaller than $T$ on each $\Sigma_{c}$ ), can be obtained by the use of equivalent Morse Theory or of Cohomological Index methods $[2,6]$. We shall not discuss this point here, and refer to [6] for an extensive analysis.

Remark 2.3. Consider the case in which $Y_{\varepsilon}$ is a Hamiltonian vector field. Denoting by $J$ the standard symplectic map, one will have

$$
Y_{\varepsilon}(z)=J d H \quad H(z)=H_{2}(z)+\varepsilon H^{\prime}(z, \varepsilon),
$$

where $\mathrm{JdH}_{2}(z)=\mathrm{Az}$ and $\left\{\mathrm{H}^{\prime}, \mathrm{H}_{2}\right\}_{\text {P.B. }}=0$.

Condition (2.5) becomes now

$$
\lambda d H_{2}(z)=d H^{\prime}(z, \varepsilon),
$$

i.e. that $z$ be a critical point of $H^{\prime}$ on a surface $\Gamma_{c} \equiv\left\{z \mid H_{2}(z)=c\right\}$, or equivalently that $z$ be a critical point of $H_{2}$ on a surface $\Gamma_{c}^{\prime} \equiv\left\{z \mid H^{\prime}(z)=c\right\}$. Notice that $t \rightarrow \exp \{A t\}$ defines a natural $S^{1}$ action on $J d H^{\prime}$ and on $\Gamma_{c}$, and also on $J d H_{0}$ and on $\Gamma_{c}^{\prime}$. Therefore Equivalent Morse theory or a Cohomological index can be used to give a lower bound on the number of periodic solutions of (2.1), with minimal period smaller than $T$, on each surface $\Gamma_{c}$ of $\Gamma_{c}^{\prime}$. In particular, at least $n$ such periodic solutions can be found if $\Gamma_{c}$ or $\Gamma_{c}^{\prime}$ are convex. It should be noted that in the former case $H_{0}$ is of definite sign [2], but that the condition that $\Gamma_{c}^{\prime}$ be convex 
poses no restriction of convexity on the surfaces

for $\varepsilon$ small.

$$
\Sigma_{c}^{\prime} \equiv\left\{z \mid H_{0}(z)+\varepsilon H^{\prime}(z)=c\right\}
$$

More generally, it is easy to see that, if one can find a real valued function $F(x, y)$ and a real constant $c$ such that

$$
\begin{gathered}
\Phi_{c} \equiv\left\{z \mid F\left(H_{0}(z), H^{\prime}(z)\right)=c\right\} \quad \text { is convex } \\
\Phi_{c} \in \mathbf{B}_{a(b)}
\end{gathered}
$$

and if $F$ does not vanish on $\Phi_{c}$, then the system (2.4) has on $\Phi_{c}$ at least $n$ periodic solutions with minimal period less than $T$ [7].

We determine now the stability of the periodic solutions described in Lemma 2.1. One has

Lemma 2.4. The Floquet multipliers $\left\{\rho_{i}, i=1 \cdots n\right\}$ for the periodic solutions described in Lemma 2.1 satisfy

$$
\left(\rho_{i}\right)^{K}=\exp \left[K \mu_{i} T_{\varepsilon}\left(z^{*}\right)\right],
$$

where $\mu_{i}$ are the eigenvalues of the matrix

$$
D G\left(z^{*}, \varepsilon\right)-\lambda\left(z^{*}, \varepsilon\right) A
$$

and $K$ is defined in (2.8). In particular (see (2.16)), if $T_{0}\left(z^{*}\right)=\underline{\tau}$, then (2.21) holds with $K=1$.

Proof. Let $p(t)$ be a periodic solution of (2.4) of the type described in Lemma 2.1; one has

$$
p(t)=\exp \{(1+\varepsilon \lambda) A t\} p(0) .
$$

The Floquet multipliers are the eigenvalues of the map

$$
R^{2 n} \ni \zeta \rightarrow \sigma\left(T_{\varepsilon}(p(0)), \zeta\right)
$$

where $\sigma(t, \zeta)$ is the solution of

$$
d \sigma / d t=\left(D Y_{\varepsilon}\right)(p(t)) \sigma, \quad \sigma(0, \zeta)=0
$$

Define $\chi(t, \zeta)$ by

$$
\chi(t, \zeta) \equiv \exp \{-(1+\varepsilon \lambda) A t\} \sigma(t, \zeta)
$$

By construction

$$
\exp \left\{K(1+\varepsilon \lambda) A T_{\varepsilon}(p(0))\right\}=I,
$$

and therefore

$$
\chi\left(K T_{\varepsilon}(p(0)), \zeta\right)=\sigma\left(K T_{\varepsilon}(p(0)), \zeta\right) .
$$

Moreover

$$
d \chi / d t=\varepsilon[D G(p(0), \varepsilon)-\lambda A] \chi,
$$


since $G$ commutes with $A z$, and therefore

$$
\exp \{-(1+\varepsilon \lambda) A t\} D G(p(t), \varepsilon)=D G(p(0), \varepsilon) \exp \{-(1+\varepsilon \lambda) A t\} .
$$

The conclusion of Lemma 2.4 follows immediately from (2.26), (2.27).

A special important case is the one in which the vector field $Y_{\varepsilon}(z)$ is Hamiltonian, so that it can be written in the form (2.18). One has then

$$
d \chi / d t=D J\left[d H^{\prime}-\lambda d H_{0}\right] \chi
$$

The vectors $\nabla H_{0}$ and $J d H_{0}$ are eigenvectors of the matrix $D J\left[d H^{\prime}-\lambda d H_{0}\right]$ with eigenvalue zero. Define $D_{0} J d H^{\prime}$ as follows:

Definition 2.5. $D_{0} J d H^{\prime}$ is the restriction of $D J d H^{\prime}$ to the orthogonal complement in $R^{2 n}$ of the subspace generated by $\nabla H_{0}$ and $J d H_{0}$.

One has then

Corollary 2.6. If the vector field has the form (2.18), and $p(t)$ is a periodic solution of (2.4) for \& sufficiently small (as described in Lemma 2.1), then $p(t)$ is elliptic precisely if all the eigenvalues of $D_{0} J d H^{\prime}$ are purely imaginary, while $p(t)$ is hyperbolic if all the eigenvalues of $D_{0} J d H^{\prime}$ have non-zero real part.

Consider now a vector field of the form

$$
Y_{\varepsilon}(z)=A z+\varepsilon G(z, \varepsilon)+\varepsilon^{p+\delta} R(z, \varepsilon)
$$

where $A$ and $G$ are as in Lemma $2.1, p \geqq 1, \delta>0$ and $R$ is uniformly bounded for $z \in \mathbf{B}_{a}, 0<\varepsilon<\varepsilon_{0}$ and Lipshitz continuous in $z$, uniformly for $z \in \mathbf{B}_{a}$ and $0<\varepsilon<\varepsilon_{0}$.

One can follow the same steps as in Lemma 2.1 and prove the following: Given $T>0$, one can find $\varepsilon_{1}>$ such that, if $\varepsilon<\varepsilon_{1}$ and $\phi\left(t, z_{\varepsilon}^{*}\right)$ is a solution of

$$
d \phi / d t=A \phi+\varepsilon G(\phi, \varepsilon)+\varepsilon^{p+\delta} R(\phi, \varepsilon), \quad \phi\left(0, z_{\varepsilon}^{*}\right)=z_{\varepsilon}^{*},
$$

then one can find $c \in R$ and $\lambda \in R$ such that

$$
\left|\lambda A z^{*}-G\left(z_{\varepsilon}^{*}, \varepsilon\right)\right|<c \varepsilon^{\delta+p-1} .
$$

Under suitable assumptions on the Jacobian of the map

$$
z^{*} \rightarrow A z^{*}+G\left(z^{*}, \varepsilon\right)
$$

(essentially that its inverse be bounded by $c_{5} \varepsilon^{-p+1}$ uniformly for $\varepsilon<\varepsilon_{1}$ and $z$ in a neighborhood of $z_{\varepsilon}^{*}$ ), condition $(2.30)$ is necessary and sufficient in order that there exist $c_{6} \in R$ and, for each $\varepsilon<\varepsilon_{1}$, a point $y_{\varepsilon}^{*} \in R^{2 n}$ such that

$$
\left|y_{\varepsilon}^{*}-z_{\varepsilon}^{*}\right|<c_{6} \varepsilon^{\delta}
$$

and $\phi_{\varepsilon}\left(t, y^{*}\right)$ is a periodic solution of (2.4) with minimal period approximately equal to $T_{\varepsilon}\left(z^{*}\right)$.

Also, results similar to those of Lemma 2.4 can be obtained on the linear stability of the periodic solutions $\phi_{\varepsilon}\left(t, y_{\varepsilon}^{*}\right)$. We shall study this problem in the next section, restricting ourselves to the case in which $Y_{\varepsilon}$ is a Hamiltonian vector field. 


\section{Applications to Hamiltonian Systems}

We use the results of Sect. 2 to study periodic solutions near equilibrium for Hamiltonian systems when the frequencies are at or near resonance. We consider a system for which the Hamiltonian $H$ has the form

$$
H(z)=1 / 2 \sum_{0}^{n} v_{i} z_{i}^{2}+H^{*}(z), \quad z=\left\{z_{1} \cdots z_{n}\right\}, \quad z_{i}=\left\{q_{i}, p_{i}\right\},
$$

where $H^{*}$ is of class $C^{p}$ in a neighborhood of the origin, for some $p \geqq 3$, and is infinitesimal at the origin of order $q \geqq 3$. We also assume that $v_{k} \neq 0$ for all $k$.

Since we are interested in the behaviour near the origin, we introduce the relevant scale through the canonical transformation

$$
z_{k} \rightarrow \varepsilon z_{k}, \quad H(z) \rightarrow \varepsilon^{-2} H(\varepsilon z) \equiv H_{\varepsilon}(z) .
$$

In the new, variables, Hamilton's equations read:

$$
d z / d t=J d H_{\varepsilon}(z)=J d H_{2}(z)+\varepsilon^{q-2} J d Q(z, \varepsilon),
$$

where

$$
H_{2}(z)=1 / 2 \sum_{1}^{n} v_{k} z_{k}^{2}, \quad Q(z, \varepsilon)=\varepsilon^{-q} H^{*}(\varepsilon z) .
$$

By construction, $Q(z, \varepsilon)$ is of class $C^{p}$ in $z$ in a ball $\mathbf{B}_{a}$, uniformly in $\varepsilon$ near 0 . We are interested in the case when the frequencies are at or near resonance. We set therefore

$$
v_{k}=v_{k}^{0}+\varepsilon^{\alpha} \mu_{k}, \quad k=1 \cdots n, \quad \mu_{k} \in \mathbf{R},
$$

where $\left(v_{k}^{0}\right\}$ is a set of resonating frequencies and the constant $\alpha$ depends on $H^{*}$ and will be chosen later.

Remark 3.0. Using the Inverse Function Theorem it is not difficult to verify [1] that for any given $T>0$ one can find positive constants $c_{1}$ and $c_{2}$ and $\varepsilon_{1}$ such that, if $|z|=a$ and $\varepsilon<\varepsilon_{1}$, and $\varphi_{\varepsilon}(t, z)$ is periodic solution of (3.3) with minimal period $T_{\varepsilon}<T$, then one must have

$$
\left|T_{\varepsilon}-N_{k} \tau\right|<c_{2} \text { for some } k \in\{1,, n\} \text { and } N_{k} \in Z,
$$

where $\tau$ is defined in $(2.3)_{1}$. Moreover in the study of periodic solutions with minimal period approximately equal to $M \tau, M \in \mathbf{Z}$, one can without loss of generality set $z_{h}=0$ if $(2 \pi)^{-1} M v_{h} \tau \notin \mathbf{Z}$. We can therefore restrict attention to the case of full resonance, i.e.

$$
v_{k}^{0}=n_{k} \underline{\underline{\nu}}, \quad n_{k} \in \mathbf{Z} /\{0\} \quad \underline{v} \neq 0 .
$$

We shall assume (3.7) from now on. Using (3.7) we write (3.3) as

$$
\begin{aligned}
d z / d t & =J d H_{0}+1 / 2 \varepsilon^{\alpha} \sum_{1}^{n} \mu_{k} J d z_{k}^{2}+\varepsilon^{q-2} J d Q(z, \varepsilon), \\
H_{0} & =1 / 2 \sum_{1}^{n} v_{k}^{0} z_{k}^{2} .
\end{aligned}
$$

For the best choice of $\alpha$ we need a lemma on normal forms. 
Lemma 3.1 [8]. Let $H_{\varepsilon}(z)$ have the form

$$
H_{\varepsilon}(z)=H_{0}(z)+\varepsilon^{r} H(\varepsilon z), \quad z \in R^{2 n},
$$

where $\mathrm{H}_{0}$ is given in $(3.8)_{2}$ and $H^{\prime}(y)$ is of class $C^{s}, s \geqq 3$, uniformly in a neighborhood of the origin, and is infinitesimal at the origin of order $d$, with $r+1 \leqq d$ and $d \leqq s$. If (3.7) holds, given $a>0$, one can find $\varepsilon_{1}>0$ such that, for each $0 \leqq \varepsilon<\varepsilon_{1}$ and each $m \leqq s-2$ there exists a symplectic transformation $\Phi_{m, \varepsilon}$, asymptotic to the identity when $\varepsilon \rightarrow 0$, and such that in $\mathbf{B}_{a}^{2 n}$,

$$
\left(H_{\varepsilon} \Phi_{m, \varepsilon}\right)(z)=H_{0}(z)+\varepsilon^{r(m)} N_{m, \varepsilon}(z)+\varepsilon^{d-r+m} R_{m, \varepsilon}(Z),
$$

where $d-r+m \geqq r(m) \geqq r(m-1), r(0)=d-r$, and $R_{m, \varepsilon}$ is infinitesimal in $\varepsilon$ uniformly for $z \in B_{a}^{2 n}$. The functions $N_{m, \varepsilon}$ and $R_{m, \varepsilon}$ are of class $C^{s-m}$ and one has

$$
\left\{N_{m, \varepsilon}, H_{0}\right\}_{\text {P.в. }}=0 .
$$

Moreover if $N_{m, \varepsilon} \neq 0$, then $r\left(m^{\prime}\right)=r(m)$ for all $m^{\prime}>m$. The function $N_{m, \varepsilon}$ will be called "normal form of $H$ to order $m$ " (relative to $H_{0}$ ).

Outline of proof. The symplectic transformation $\Phi_{m, \varepsilon}$ is constructed in $m$ steps. At step $i$ the transformation is the time- $\varepsilon$ map $\Phi^{(i)}$ for a suitable Hamiltonian $K_{i}$. To find $K_{i}$ one has to solve

$$
\left\{K_{i}, H_{0}\right\}_{\text {P.B. }}=B_{i-1} \text {, }
$$

where $B_{i-1}$ is known from step $i-1$ and has no component in the kernel of the map $F \rightarrow\left\{F, H_{0}\right\}_{\text {P.в. }}$. No small denominator arises, since by (3.7), if $\sum_{1}^{n} m_{k} v_{k} \neq 0$, $m_{k} \in Z$, then $\left|\sum_{1}^{n} m_{k} v_{k}\right|>\underline{v}$. On the other hand, at each step one loses in general one order of differentiability, since the map $\Phi^{(i)}$ and the functions $B_{i}$ are as regular as $J d K_{i}$, while the solution $K_{i}$ of (3.12) has one order of differentiability less than $B_{i-1}$.

We now apply Lemma 3.1 to the study of periodic solutions of (3.8). Notice that the term

$$
1 / 2 \sum_{1}^{n} \mu_{k} z_{k}^{2}
$$

is in normal form with respect to $H_{0}$, and recall that

$$
\{F, G\}_{\text {P.B. }}=0 \Rightarrow[J d F, i / l i]_{\text {Lie }}=0 .
$$

Consider the Hamiltonian

$$
H_{\varepsilon}^{\prime}(z) \equiv H_{0}(z)+\varepsilon^{q-2} Q(z, \varepsilon) .
$$

By construction $Q(z, \varepsilon)$ satisfies the assumptions of Lemma 3.1 with $r=2, d=q$, $s=p$. For every $m \leqq p$ one can therefore write $H^{\prime}$ in new symplectic coordinates (still denoted by $z$ ) in the form

$$
H_{\varepsilon}^{\prime}(z)=H_{0}(z)+\varepsilon^{r(m)} N_{m, \varepsilon}^{\prime}(z)+\varepsilon^{d(m)} R_{m, \varepsilon}^{\prime}(z)
$$


where $r(m) \geqq q-2, d(m) \geqq q-2+m, N_{m, \varepsilon}^{\prime}$ satisfies (3.11) and $R_{m, \varepsilon}^{\prime}$ is infinitesimal in $\varepsilon$ uniformly for $z \in \mathbf{B}_{a}$. We consider only the case in which one can find $m \leqq p$ such that $N_{m}^{\prime} \neq 0$ (while $N_{m}^{\prime}=0$ if $m<\underline{m}$ ), and choose $\alpha$ in (3.5) as

$$
\alpha=r(\underline{m}) \text {. }
$$

One has then, using Lemma 3.1, for all $\underline{m} \leqq m \leqq p$,

$$
H_{\varepsilon}(z)=H_{0}(z)+\varepsilon^{\alpha}\left[1 / 2 \sum_{1}^{n} \mu_{k} z_{k}^{2}+N_{m, \varepsilon, \mu}(z)\right]+\varepsilon^{d(m)} R_{m, \varepsilon, \mu}(z),
$$

where the $N_{m, \varepsilon, \mu}$ satisfy (3.11), and $d(m) \geqq q-2+m, d(m) \geqq \alpha$ and $d(m)>\alpha$ if $m>\underline{m}$. The functions $R_{m, \varepsilon, \mu}$ are infinitesimal in $\varepsilon$ uniformly for $z \in \mathbf{B}_{a}$. The best choice of $m, \underline{m} \leqq m \leqq p$, depends on the resonant set of frequencies. This will be seen explicitly in the example we shall discuss in the next section.

Define the Hamiltonians $\underline{H}_{\varepsilon}$ by

$$
\underline{H}_{\varepsilon}(z)=H_{0}(z)+\varepsilon^{\alpha}\left[1 / 2 \sum_{1}^{n} \mu_{k} z_{k}^{2}+N_{m, \varepsilon, \mu}(z)\right] .
$$

The term in square brackets in (3.16) is by construction in normal form with respect to $H_{0}$. We are therefore in condition to apply to the Hamiltonian flow of $\underline{H}_{\varepsilon}$ Lemmas 2.1 and 2.4, Remark 2.3 and Corollary 2.5.

Remark 3.2. We notice in particular that $N_{m, \varepsilon, \mu}$ has by construction the form

$$
\varepsilon^{r(m)} N_{m, \varepsilon, \mu}(z)=\sum_{s=3}^{m} \varepsilon^{s} P_{s, \mu}(z)
$$

where $P_{s, \mu}(z)$ are homogeneous polynomials of order $s$, normal with respect to $H_{0}$. Therefore the solutions $z_{\varepsilon}, \lambda_{\varepsilon}, \mu_{\varepsilon}$ of

$$
X_{m, \varepsilon, \mu}(z, T) \equiv d N_{m, \varepsilon, \mu}(z)-\lambda d H_{0}(z)=0
$$

form continuous families, with parameter $\varepsilon$.

We have proved in Sect. 2 that for any given $T>0$, for sufficiently small $\varepsilon$ the $\varphi_{0}$-orbits of solutions of (3.18) are in one-to one correspondence with the periodic solutions of

$$
d z / d t=J d \underline{H}_{\varepsilon}(z)
$$

with minimal period $\leqq T$.

We shall now study, still for $\varepsilon$ sufficiently small, the periodic solutions of (3.3) with minimal period $T_{\varepsilon} \leqq T$. When $\mu_{k}=0 \forall k$, this is equivalent to the study of periodic solutions of

$$
d z / d t=J d H(z)
$$

(with minimal period $T_{\varepsilon}(p(0)) \leqq T$ ) in a small neighborhood of the origin. When some of the $\mu_{k}$ are $\neq 0$, the problem we study can be regarded [5] as a bifurcation analysis for periodic solutions of (3.20).

Since we are interested in small values of $\varepsilon$, we shall use the Inverse Function Theorem. We make therefore an assumption on the Jacobian of the map 
$z \rightarrow X_{m, \varepsilon, \mu}\left(z, T_{\varepsilon}\right)$, with $X_{m, \varepsilon, \mu}(z, T)$ defined in (3.18) (the relation between $T$ and $\lambda$ is given in (2.11)). This assumption holds only if $p$ is large enough (depending on the choice of $v_{1}^{0} \cdots v_{n}^{0}$ ), and, for given $p$, only if $N_{m, \varepsilon, \mu}$ does not belong to a subspace of codimension greater or equal to one in the vector space of polynomials of order $\leqq m$ normal with respect to $H_{0}$. In this sense, condition (3.22) in the next proposition is satisfied generically.

Proposition 3.3. Let the Hamiltonians $H_{\varepsilon}$ have the form (3.15), with $t(m)>\alpha$ and $R_{m, \varepsilon, \mu}$ uniformly bounded for $z \in B_{a}$. Define $X_{m, \varepsilon, \mu}$ by (3.18) and denote by $\mathrm{Hess}_{0} B$ the restriction of the Hessian matrix of $B$ to the orthogonal complement in $R^{2 n}$ to the subspace spanned by $\nabla H_{0}$ and $J d H_{0}$. Then, for every $\delta<d(m), c_{k}>0, k=1,2,3$, $\mu \in R^{n}$, one can find $\varepsilon_{1}>0$ such that the following statements hold true:

I: if $\varepsilon<\varepsilon_{1}$ and $z_{\varepsilon}, T_{\varepsilon}^{0}$ is a solution of

$$
X_{m, \varepsilon, \mu}\left(z_{\varepsilon} \cdot T_{\varepsilon}^{0}\right)=0,
$$

which satisfies

$$
\left.\varepsilon^{-m} \operatorname{det}\left[\operatorname{Hess}_{0} N_{m, \varepsilon, \mu}(z)\right]\right|_{z=z_{r}}>c_{1},
$$

then one can find $y_{\varepsilon} \in R^{2 n}$ such that

a) $\left|y_{\varepsilon}-z_{\varepsilon}\right|<c_{2} \varepsilon^{\tau(m)-\delta}$.

b) $\varphi_{\varepsilon}\left(t, y_{\varepsilon}\right)$ is a periodic solution of (3.3) with period $T_{\varepsilon}\left(y_{\varepsilon}\right)$ approximately equal to the minimal period $T_{0, \varepsilon}$ of $\varphi_{0}\left(t, z_{\varepsilon}\right)$.

c) $\Delta\left(\varphi_{\varepsilon}, \varphi_{0}\right)<c_{3} \varepsilon^{d(m)-\delta}$, where $\Delta\left(\varphi_{\varepsilon}, \varphi_{0}\right)$ is the distance of the orbits of $\varphi_{\varepsilon}\left(t, y_{\varepsilon}\right)$ and of $\varphi_{0}\left(t, z_{\varepsilon}\right)$.

The point $y_{\varepsilon}$ is not unique; however the periodic solution $\varphi_{\varepsilon}\left(t, y_{\varepsilon}\right)$ is uniquely determined by the condition

d) $H_{\varepsilon}(y)=\underline{H}_{\varepsilon}(z)$

$\left(H_{\varepsilon}\right.$ and $\underline{H}$ are defined in (3.15), (3.16)).

II: Conversely, if $\varepsilon<\varepsilon_{1}$ and $y_{\varepsilon} \in R^{2 n}$ is such that $y_{\varepsilon}$ satisfies (3.22) and $\varphi_{\varepsilon}\left(t, y_{\varepsilon}\right)$ is periodic solution of (3.3) with minimal period $T_{\varepsilon}\left(y_{\varepsilon}\right)<T$, there exists $z_{\varepsilon} \in R^{2 n}, \lambda_{\varepsilon} \in R$ such that

a) $\left|z_{\varepsilon}-y_{\varepsilon}\right|<c_{2} \varepsilon^{d(m)-\delta}$.

b) $X_{m, \varepsilon, \mu}\left(z_{\varepsilon}\right)=0$ holds, so that $\varphi_{0}\left(t, z_{\varepsilon}\right)$ is a periodic solution of (3.19) with period $T_{0}^{\prime}$ approximately equal to $T_{\varepsilon}\left(y_{\varepsilon}\right)$.

c) $\Delta\left(\varphi_{0}\left(t, z_{\varepsilon}\right), \varphi_{\varepsilon}\left(t, y_{\varepsilon}\right)\right)<\varepsilon^{d(m)-\delta}$.

The point $z_{\varepsilon}$ is not uniquely determined; however the periodic solution $\varphi_{0}\left(t, z_{\varepsilon}\right)$ is uniquely determined by the condition $\underline{H}_{\varepsilon}\left(z_{\varepsilon}\right)=H_{\varepsilon}\left(y_{\varepsilon}\right)$.

Remark 3.4. A more careful analysis shows that if $z_{\varepsilon}$ is a solution of (3.21) and if condition (3.22) is satisfied, then $\varphi_{0}\left(t, z_{\varepsilon}\right)$ has minimal period $\underline{\tau}$, i.e. the greatest among the minimal periods of the solutions of $d z / d t=J d H_{0}$. As a consequence, $K=1$ in Lemma 2.1. Similarly, under condition (3.22), $\varphi_{\varepsilon}\left(t, y_{\varepsilon}\right)$ in Proposition 3.2, II, has minimal period $T_{\varepsilon}$ which differs from $\underline{\tau}$ by terms of order $\varepsilon^{d(m)-\delta}$.

Proof of Proposition 3.3. We prove first part I. The condition that $\varphi_{\varepsilon}\left(t, y_{\varepsilon}\right)$ be 
periodic with period $T_{\varepsilon}$ is equivalent to

$$
\Phi_{\varepsilon}\left(T_{\varepsilon}, y_{\varepsilon}\right)=\int_{0}^{T \varepsilon} J d H_{\varepsilon}\left(\varphi_{\varepsilon}(t, y)\right) d t=0
$$

Since $H_{\varepsilon}$ is constant along the flow of $J d H_{\varepsilon}$, it is sufficient to consider the restriction of (3.24) to

$$
(y, T) \in \Sigma_{\varepsilon} \times R,
$$

where $\Sigma_{\varepsilon} \equiv\left\{z \mid H_{\varepsilon}(z)=H_{\varepsilon}\left(y_{\varepsilon}\right)\right\}$. Moreover, since $H_{\varepsilon}$ does not depend on time, it is sufficient to solve (3.24) on a Poincarè section at $y_{\varepsilon}$, in particular on the hyperplane perpendicular to $J d H_{\varepsilon}$. Proceeding as in the proof of Lemma 2.1 and using the fact that

$$
\varphi_{\varepsilon}(s, y)-\underline{\varphi}_{\varepsilon}(s, y)=0\left(\varepsilon^{d(m)}\right)
$$

the restricted condition can be written as

$$
\Theta^{\prime}(y, T) \equiv X_{m, \varepsilon, \mu}^{\prime}(y, T)+\varepsilon^{d(m)} R_{m, \varepsilon, \mu}^{\prime}(y, T)=0,
$$

where $R^{\prime}$ is uniformly bounded in the domain considered, and $A^{\prime}$ is the restriction of $A$ to the orthogonal complement to the subspace $\Xi_{\varepsilon}$ spanned by $J d H_{\varepsilon}$ and $\nabla H_{\varepsilon}$.

By assumption (3.22), the Jacobian of the map $y \rightarrow \Theta(y, T)$ is non-singular for $\varepsilon<\varepsilon_{1}$. Moreover $d \Phi(y, T) / d T=J d H_{\varepsilon}(y) \neq 0$, so that also the Jacobian of the map $y, T \rightarrow \Theta^{\prime}(y, T), T$ is not singular. By assumption, $z_{\varepsilon}, \underline{T}_{\varepsilon}$ solve the equation

$$
X_{m, \varepsilon, v}^{\prime \prime}(z, T)=0
$$

where $X^{\prime \prime}$ is the restriction of $X$ to the orthogonal complement to the subspace $\Xi_{\varepsilon}$ generated by $J d \underline{H}_{\varepsilon}$ and $\nabla \underline{H}_{\varepsilon}$. Since $H_{\varepsilon}-\underline{H}_{\varepsilon}=0\left(\varepsilon^{d(m)}\right)$, there exists an orthogonal transformation $\Psi_{\varepsilon}$ which is of the form $I+0\left(\varepsilon^{d(m)}\right)$ and maps $\Xi_{\varepsilon}$ onto $\Xi_{\varepsilon}$. Part I, a), b), c), d) are then a consequence of the Implicit Function Theorem.

To prove Part II, one follows the same steps. Given a solution $\{y, T\}$ of (3.25), one can write (3.26) in the equivalent form

$$
0=X^{\prime \prime}(z, T) \equiv \Theta^{\prime}(z, T)+\varepsilon^{d(m)} R^{\prime \prime}(z, T),
$$

where $R^{\prime \prime}$ is defined by (3.27). Since $\{y, T\}$ solves $\Theta^{\prime}(z, T)=0$, Part II, a), b), c), follows from the Inverse Function Theorem in view of assumption (3.22).

The stability properties of the solutions of (3.3) described in Proposition 3.3 follow immediately from Corollary 2.6. One has in particular

Corollary 3.5. The solution $\varphi_{\varepsilon}\left(t, y_{\varepsilon}\right)$ of (3.3) described in Proposition 3.3 is hyperbolic if all the eigenvalues of $D_{0} J d \underline{H}_{\varepsilon}\left(z_{\varepsilon}\right)$ have non-zero real part. It is elliptic if all eigenvalues of $D_{0} J d \underline{H}_{\varepsilon}\left(z_{\varepsilon}\right)$ are purely imaginary and have multiplicity one.

\section{Applications: The Cases $n=2, \phi_{2}^{0}= \pm 2 \phi_{1}^{0}, \phi_{2}^{0}= \pm 3 / 2 \phi_{1}^{0}$}

In this section we apply the formalism of Sect. 3 to three simple examples. A partial analysis of the first and the second have been given previously [3,4] (for an analysis of the detuning within a different approach, see e.g. [5]). We present them here 
to illustrate the general formalism. The third example is discussed because it illustrates generic properties of systems for which $n=2, v_{1}^{0}=m_{1} \underline{v}, v_{2}^{0}=m_{2} \underline{v}$, $\left|m_{1}\right|+\left|m_{2}\right| \geqq 5$. The common feature of these systems is that generically $\mathrm{H}-\mathrm{H}_{2}$ has a non-trivial normal form $N_{4}$ of order four. However $N_{4}$ is invariant under a one-parameter group of transformations different from the one generated by $J d H_{0}$, so that $D_{0} J d N_{4}$ is singular (it is in Jordan form and has zero as eigenvalue with algebraic multiplicity 2).

In order to apply the results of Sect. 3, one must assume more regularity and construct the normal form of $H$ to the order $\left|m_{1}\right|+\left|m_{2}\right|$ (which is the first order in which this continuous symmetry is broken). We refer to [9] for other examples, in particular the cases $n=2,\left|v_{2}\right|=k v_{1}, k \geqq 4$ and $n=3,\left|v_{3}\right|=2\left|v_{2}\right|=4 v_{1}$; $n=3\left|v_{2}\right|=2\left|v_{1}\right|$ (see also [4]).

We begin now the analysis of the examples mentioned above.

A) $v_{2}^{0}=2 v_{1}^{0}$.

We shall only discuss periodic solutions which have minimal period approximately equal to $\tau_{1}^{0} \equiv \underline{\tau}$. The periodic solutions which have minimal period approximately equal to $\tau \equiv \tau_{2}^{0}$ form a one-parameter family which depends smoothly on $\mu_{1}$ and $\mu_{2}$. This follows from Remark 3.0.

We assume that $H$ is at the origin of class $C^{3+\sigma}, \sigma>0$; by a change in time scale we can set $v_{1}^{0}=1, \mu_{1}=0$. We write $\mu$ for $\mu_{2}$. After the symplectic transformation described in Sect. 3, and the scaling $z \rightarrow \varepsilon z$, the Hamiltonians $H_{\varepsilon}$ have the form

$$
H_{\varepsilon}=1 / 2\left(p_{1}^{2}+q_{1}^{2}\right)+\left(p_{2}^{2}+q_{2}^{2}\right)+\varepsilon N\left(z_{1}, z_{2}\right)+\varepsilon^{1+\delta} R\left(z_{1}, z_{2}, \varepsilon\right)
$$

where $z_{i}=\left\{p_{i}, q_{i}\right), \delta>0$ and

$$
N\left(z_{1}, z_{2}\right)=a\left[\left(p_{1}^{2}-q_{1}^{2}\right) p_{2}+2 p_{1} q_{1} q_{2}\right]+\mu\left(p_{2}^{2}+q_{2}^{2}\right) .
$$

Remark. In the notation $\zeta_{k} \equiv p_{k}+i q_{k}$, the flow of $J d H_{0}$ is given by

$$
\zeta_{1}(t)=\zeta_{1}(0) \exp \left\{i v_{1} t\right\}, \quad \zeta_{2}(t)=\zeta_{2}(0) \exp \left\{i v_{2} t\right\}
$$

so that $\zeta_{1}^{* 2} \zeta_{2}$ and $\zeta_{1}^{2} \zeta_{2}^{*}$ are the only monomials of order 3 which are invariant under the flow of $J d H_{0}$. Changing the phase of $\zeta_{1}$ and $\zeta_{2}$ is a symplectic transformation; one can therefore choose symplectic coordinates such that $N\left(z_{1}, z_{2}\right)$ has the form given above.

We assume that $a \neq 0$ in (4.2); without loss of generality one can then take $a=1$. (If $a=0$, one is in the framework of example 3 below.) Since normal forms to order 3 coincide with time-averages along the flow of $J d H_{0}$, this assumption holds for all the Hamiltonians $\mathrm{H}$ in $\mathrm{C}^{3}$ for which the time average of $\mathrm{H}-\mathrm{H}_{2}$ is not infinitesimal at the origin of order greater than three. In this sense our results are generic.

One is now condition to use the results of Sect. 3. One has $q=3, m_{0}=1$, $r\left(m_{0}\right)=1$ and in (4.2) we have already taken $\alpha=1$. One can choose as Poincaré section the hyperplane $q_{1}=0$. The condition

$$
d N=\lambda d H_{0}
$$




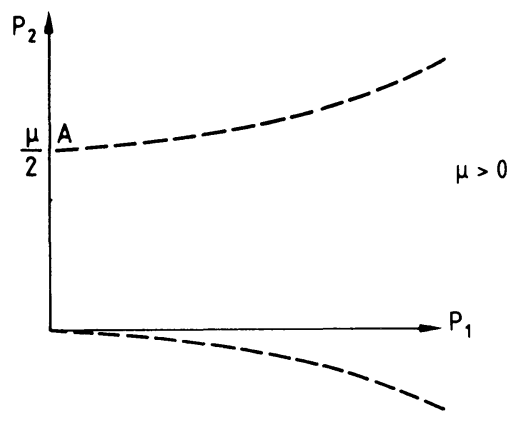

Fig. 1

becomes then

$$
2 p_{1} p_{2}=\lambda p_{1}, \quad p_{1}^{2}+2 \mu p_{2}=2 \lambda p_{2}, \quad q_{2}=0 .
$$

We consider only solutions for which $p_{1} \neq 0$; the other solution corresponds to the family of periodic solutions with period approximately equal to $\tau_{2}^{0}$, which is obtained in Lyapunov's analysis. Also, only the case $p_{1}>0$ will be considered, since $\left\{-z_{1}^{0}, z_{2}^{0}\right\}$ belongs to the orbit of the periodic solution of $d z / d t=J d H_{0}$ with initial data $\left\{z_{1}^{0}, z_{2}^{0}\right\}$. Since $p_{1} \neq 0$, one has $K=1$ in Lemma 3.4. One has then

$$
\lambda^{ \pm}=2 p_{2} \quad p_{2}^{ \pm}=1 / 4\left[\mu \pm\left(\mu^{2}+4 p_{1}^{2}\right)^{1 / 2}\right] .
$$

The graph of $p_{2}^{ \pm}\left(p_{1}\right)$ is given in Fig. 1 for fixed $\mu>0$. It is also straightforward to compute the spectrum of $D J\left(d N-\lambda d H_{0}\right)$.

For both alternatives \pm in (4.5), one finds that, apart from the eigenvalue zero which has multiplicity two, there are the two eigenvalues

$$
\sigma_{ \pm}\left(p_{1}\right)=i\left|p_{1}\right|\left|\left[p_{1}^{2}+8 p_{2}^{2}\right]^{1 / 2} /\right| p_{2} \mid \text {. }
$$

In particular $\left|\left(D_{0} J d N\right)^{-1}\right|=1 /\left|\sigma\left(p_{1}\right)\right|$, so that $D_{0} J d N$ is not singular at the solution of (4.3), with the exception of the point $A$ in Fig. 1.

We conclude from Proposition 3.3 that, given $a>0$, for every sufficiently small $\varepsilon$ there are two periodic solutions of (3.3) with period approximately equal to $\tau_{1}^{0}$ and orbit approximately equal to the orbit of the flow of $J d H_{0}$ through the point

$$
p_{1}=a, \quad q_{1}=0, \quad q_{2}=0 \text { and } \quad p_{2}^{ \pm} \quad \text { as in (4.5). }
$$

From (4.6) and Corollary 3.4 it follows that these periodic solutions are elliptic. Notice that the point $A$ in Fig. 1 is a bifurcation point with period doubling for the periodic solutions of $d z / d t=J d \underline{H}_{\varepsilon}$, but the same conclusion cannot be drawn in general for the periodic solutions of (3.3), since condition (3.22) fails at the point $A$.

Remark. For $|\mu|$ large, one of the two solutions in (4.5) (which one depends on the sign of $\mu$ ) is such that $\left|p_{2}\right|$ becomes very small (see Fig. 1). This corresponds to the periodic solution which is found in Lyapunov analysis; in fact, large $\mu$ corresponds to a detuning which is large compared to the size of the neighborhood of the origin in $R^{2 n}$ in which one looks for periodic solutions, and corresponds therefore to a non-resonant set of frequencies. This can be explicitly seen if one 
makes a different rescaling of the detuning term, i.e. writes $v_{k}=v_{k}^{0}+\varepsilon^{\alpha} \mu_{k}$, with $\alpha<1$. On this scale, the norm of the other solution in (4.5) becomes large when $\varepsilon$ decreases, and therefore when $\varepsilon$ is very small the Implicit Function Theorem cannot be used to deduce the existence of a corresponding periodic solution of (3.3). As a consequence, in this scaling of the detuning term, in a sufficiently small neighborhood of the origin there is in general only one family of periodic solutions with minimal period approximately equal to $\tau_{1}^{0}$, in accordance with Lyapunov' analysis.

B) $v_{2}^{0}=-2 v_{1}^{0}$.

Also in this case we shall discuss only the periodic solutions which have minimal period approximately equal to $\tau_{1}^{0}$. The Hamiltonians $H_{\varepsilon}$ take now the form (4.1), where

$$
N\left(z_{1}, z\right)=\left(p_{1}^{2}-q_{1}^{2}\right) p_{2}-2 p_{1} q_{1} q_{2}+\left(p_{2}^{2}+q_{2}^{2}\right) .
$$

Choosing the same Poincare section as in the previous example, condition (4.2) becomes now

$$
2 p_{1} p_{2}=p_{1}, \quad p_{1}^{2}+2 p_{2}=-2 p_{2}, \quad q_{2}=0, \quad q_{1}=0 .
$$

Again we consider only the solutions for which $p_{1} \neq 0$. One has then

$$
\lambda=2 p_{2}, \quad p_{2}^{ \pm}=1 / 4\left[-\mu \pm\left(\mu^{2}-4 p_{1}^{2}\right)^{1 / 2}\right] .
$$

From (4.10) one can see that solutions exist only if $2 p_{1} \leqq|\mu|$; in particular there is no solution if $\mu=0$, i.e. in the resonant case. It is also seen from (4.10) that for each $\mu>0$ the solutions lie on a curve in the $p_{1} p_{2}$ plane, parametrized by $p_{2}$ (see Fig. 2); as in the previous example, only the half-plane $p_{1}>0$ must be considered. The energy cannot be used as parameter (see Fig. 3).

To study the stability of those periodic solutions of (3.3) which correspond to the solutions (4.10) of (4.9), we make use of Corollary 3.4 and compute the spectrum of $D J\left(d N-d H_{0}\right)$. Apart from the eigenvalue zero, one finds the eigenvalues

$$
\sigma^{ \pm}= \pm\left|p_{1} p_{2}^{-1}\right|\left(8 p_{2}^{2}-p_{1}^{2}\right)^{1 / 2} \text {. }
$$

Condition (3.22) is satisfied along the curve in Fig. 1, with the exception of the point $A$ and $B$. Therefore those solutions for which $8 p_{2}^{2}<p_{1}^{2}$, i.e. the part of the

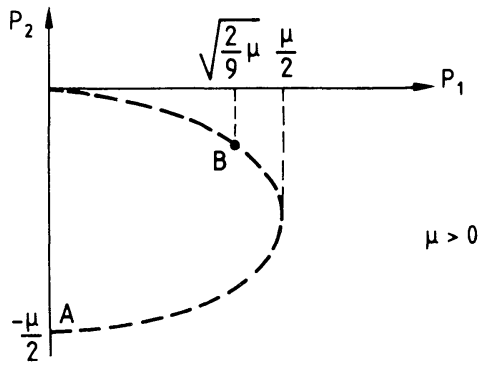

Fig. 2 


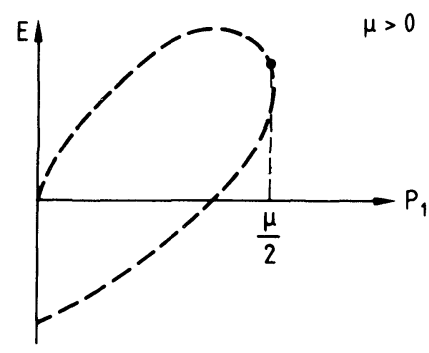

Fig. 3

upper branch of the curve in Fig. 2 which lies to the left of the point B, correspond to elliptic periodic solutions, while the part of the curve in Fig. 2 which starts at the right of the point $B$ corresponds to periodic solutions which are hyperbolic. When $8 p_{2}^{2}=p_{1}^{2}$, the matrix $D_{0} J d N$ is singular, and Proposition 3.3 cannot be used. To study this case, one should make the further assumption that $H$ is of class $C^{4}$ at the origin, and construct the normal form of $H$ to order four.

Remark. For $\mu$ very large, $p_{2}$ becomes very small compared to $p_{1}$ on the elliptic branch of solutions, at least for values for $p_{1}$ small compared to $\mu$. This branch of solutions goes over, when $\mu$ is large, to the family of solutions found in Lyapunov's analysis for the strongly detuned case, i.e. the case in which the two frequencies $v_{1}$ and $v_{2}$ are not in resonance. This is seen explicitly if one does a different rescaling of the detuning term in (4.2).

C) $v_{2}^{0}= \pm 3 / 2 v_{1}^{0}$.

It follows from Lyapunov's analysis that, for $i=1,2$, there exists a one-parameter family of periodic solutions, with period approximately equal to $\tau_{i}^{0}$ and lying approximately on the hyperplane $z_{j}=0, j \neq i$. We are interested in periodic solutions with minimal period longer than the one of Lyapunov's solutions. By a change in time scale, we can take $v_{1}^{0}=2, v_{2}^{0}= \pm 3$, which corresponds to the choice $\underline{v}=1$ in (3.7). As in the previous examples, we can also take $\mu_{1}=0$ and write $\mu$ for $\mu_{2}$. In the present case, in order to satisfy (3.22) — at least generally - one must assume that $H$ is of class $C^{4+\delta}$ at the origin, $\delta>0$, and take $m=2$, so that $d(m)=2+\delta$. The Hamiltonians $H_{\varepsilon}$ can then be written in the form (4.1) where, for $v_{2}^{0}=3$ one has

$$
\begin{aligned}
N\left(z_{1}, z_{2}, \varepsilon\right)= & c_{1}\left(p_{1}^{2}+q_{1}^{2}\right)^{2}+c_{2}\left(p_{2}^{2}+q_{2}^{2}\right)^{2}+\mu\left(p_{2}^{2}+q_{2}^{2}\right) \\
& +\varepsilon c_{3}\left[p_{1}\left(p_{1}^{2}-3 q_{1}^{2}\right)\left(p_{2}^{2}-q_{2}^{2}\right)-2 q_{1} q_{2} p_{2}\left(q_{1}^{2}-3 p_{1}^{2}\right)\right],
\end{aligned}
$$

while for $v_{2}^{0}=-3$ the second term in the square bracket has opposite sign. We shall assume that the constants $c_{1}, c_{2}, c_{3}$ are all different from zero. (This condition holds generically for Hamiltonians of class $C^{4}$.) Without loss of generality one can then take $c_{3}=1$.

Making use of the same Poincaré section as in the previous two examples, condition (4.2) becomes

$$
\begin{aligned}
q_{1}= & q_{2}=0, \quad 4 c_{1} p_{1}^{3}+3 p_{1}^{3} p_{2}^{2}=2 \lambda p_{1}, \\
& 4 c_{2} p_{2}^{3}+2 \mu p_{2}+2 \varepsilon p_{1}^{3} p_{2}= \pm 3 \lambda p_{2} .
\end{aligned}
$$


We consider only solutions of (4.12) for which $p_{1} \neq 0$ and $p_{2} \neq 0$. Solutions which do not satisfy these two conditions correspond to the periodic solutions which are obtained through Lyapunov's analysis.

Since $\left\{z_{1}^{0},-z_{2}^{0}\right\}$ and $\left\{z_{1}^{0}, z_{2}^{0}\right\}$ belong to the same orbit of $d z / d t=J d H_{0}$, we must consider only those solutions of (4.12) for which $p_{2}>0$. From (4.12) one derives

$$
-p_{2}^{2}\left(-8 c_{2} \pm 9 \varepsilon p_{1}\right)= \pm p_{1}^{2}\left(12 c_{1}-4 \mu p_{1}\right)-2 \mu, \quad p_{2}>0 .
$$

Solutions of (4.13) exist for various ranges of the parameter $\mu$, depending on the relative sign of the coefficients $c_{1}$ and $c_{2}$, and of whether one chooses the + sign or the $-\operatorname{sign}$ in (4.13) (i.e. whether one considers the case $v_{2}^{0}=+3 / 2 v_{2}^{0}$ or the case $\left.v_{2}^{0}=-3 / 2 v_{1}^{0}\right)$. Since the two equations in (4.13) are interchanged if one changes sign to $c_{1}$ and $\mu$, we shall consider only the upper alternative in (4.13), i.e. the case $v_{2}^{0}=3 / 2 v_{1}^{0}$. When $\mu=0, C_{1} / c_{2}<0$. there are no solution. When $\mu=0, c_{1} / c_{2}>0$, the equation has two solutions for each value of $p_{2}$.

In Figs. 4 and 5 we illustrate the cases $c_{1}, c_{2}>0$ and $c_{1}>0, c_{2}<0$ respectively, for a fixed value $\mu>0$. The case $\mu<0$ leads to similar figures and results. At the solutions of (4.13) the matrix $D J\left(d N-d H_{0}\right)$ has, in addition to the eigenvalue zero, two eigenvalues

$$
\sigma^{ \pm}= \pm\left|p_{1} p_{2}\right|\left[\left(8 c_{2}+18 c_{1}\right) \varepsilon p_{1}+0\left(\varepsilon^{2}\right)\right]^{1 / 2} .
$$

Therefore, on every point of the curve in Fig. 4, with the exception of the points $A$ and $B$, condition (3.22) is satisfied if $\varepsilon$ is sufficiently small. The corresponding periodic solutions of (3.3) are elliptic on the branch for which $\left(8 c_{2}+18 c_{1}\right) p_{1}<0$, and hyperbolic on the other branch. If $8 c_{2}+18 c_{1} \neq 0$, the same conclusion holds for the points on the curve in Fig. 5. If the parameters in $N$ satisfy the identity $8 c_{2}=18 c_{1}$, one has to analyze in (4.14) the terms or order two in $\varepsilon$. One finds that for this value of the parameters the non-zero eigenvalues zre $\sigma^{ \pm}= \pm i 3 \sqrt{ } 2 \varepsilon\left|p_{1}^{2} p_{2}\right|$. Therefore also for this choice of parameters condition (3.22) is satisfied and, if $\varepsilon$ is sufficiently small, for every point on the curve in Fig. 5, with the exception of the points $A$ and $B$, one has periodic solutions of (3.3) which are elliptic.

Remark. One sees that in both cases, for every fixed $a>0$, solutions satisfying $\left|z_{1}^{2}\right|+z_{2}^{2} \mid \leqq a^{2}$ exist only if the parameter $\mu$ is in a range $\mu_{1}(a) \leqq \mu \leqq \mu_{2}(a)$. For every $\varepsilon>0$, if $\mu$ is sufficiently large, one is beyond the range of applicability of the

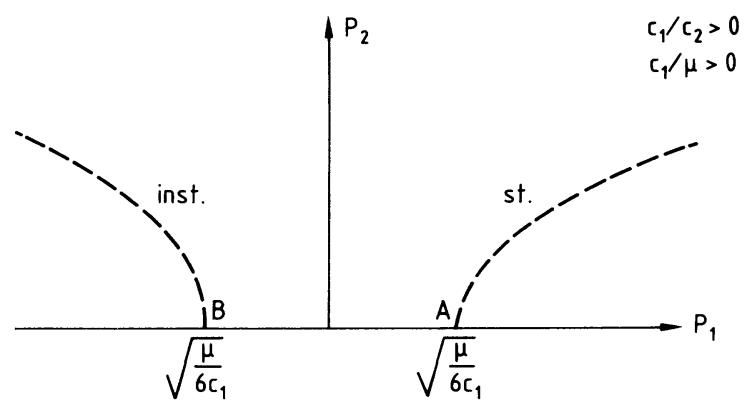

Fig. 4 


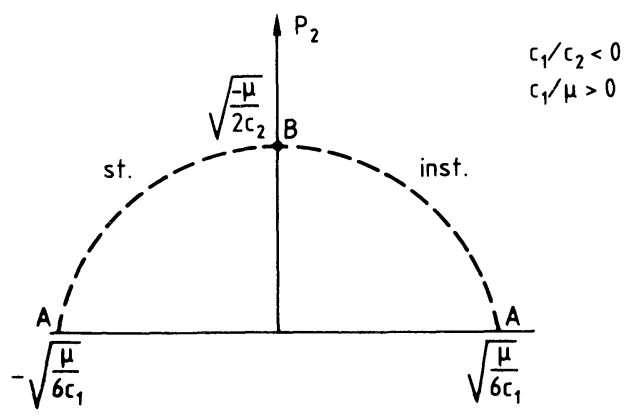

Fig. 5

Inverse Function Theorem. Therefore, in the limit of "large detuning" (i.e. when the frequencies are not in resonance) one cannot conclude in general that in a neighborhood of the origin there are periodic solutions of (3.3) with minimal period not approximately equal to either $\tau_{1}^{0}$ or $\tau_{2}^{0}$, in accordance with Lyapunov's analysis.

Acknowledgements. The author is grateful to E. Zehnder for very stimulating discussions; he is also indebted to an unknown referee for constructive criticism.

\section{References}

1. Lyapunov, A.: Probléme gèneral de la stabilité du mouvement, Ann. Math. Studies $N^{0} 17$. Prınceton, NJ: Princeton University Press 1947

2. Weinstein, A.: Normal modes for non-linear Hamiltonian Systems. Inv. Math. 20, 47-57 (1973); Moser, J.: Periodic solutions near equilibrium and a Theorem by A. Weinstein, Commun. Pure Appl. Math. 29, 727-747 (1976)

3. Schmidt, D.: Periodic solutions near a resonant equilibrium of a Hamiltonian System. Cel. Mech 9, 81-103 (1974)

4. Dell'Antonio, G. F., D’Onofrio, B.: Periodic solutions of Hamiltonian Systems near equilibrium I. Boll. UMI 6, 809-835 (1984)

5. Duistermaat, J.: Bifurcation of periodic solutions near equilibrium points of Hamiltonian systems. Lecture Notes in Mathematics, vol. 1057, pp. 57-105. Berlin, Heidelberg, New York: Springer 1986

6. Fadell, E., Rabinowitz, P.: Generalized cohomological index theories for Lie group actions with an application to Bifurcation questions for Hamiltonian Systems. Invent. Math. 45, 139-174 (1978)

7. Dell'Antonio, G. F.: An application of Topological Methods... In: Geometrodynamics Proceedings. (ed.) pp. 101-138. Prastaro, Singapore: World Scientific 1985

8. Churchill, C., Kummer, M., Rod, D.: On averaging, reduction and symmetry in Hamiltonian Systems. J. Diff. Eq. 49, 359-414 (1983)

9. Dell'Antonio, G. F.: Periodic solutions of Hamiltonian systems near equilibrium, In: Advanced topics in the theory of dynamical systems. Trento, June 1987. New York: Academic Press

Communicated by J. N. Mather

Received December 8, 1987; in revised form July 29, 1988 